\title{
Internações hospitalares por agrotóxicos: registros de uma unidade sentinela de
}

\section{assistência toxicológica}

\author{
Hospital admissions for pesticides: records of a sentinel toxicological care unit \\ Ingresos hospitalarios por plaguicidas: registros de una unidad centinela de atención toxicológica
}

Recebido: 29/01/2022 | Revisado: 03/02/2022 | Aceito: 11/02/2022 | Publicado: 17/02/2022

André Soares da Silva
ORCID: https://orcid.org/0000-0002-7231-8048
Universidade Estadual do Maringá, Brasil
E-mail: andre.7022@ @otmail.com
Daniela Aparecida de Souza Nunes
ORCID: https://orcid.org/0000-0003-4621-7208
Universidade Estadual do Maringá, Brasil
E-mail: danisouza.enf@ gmail.com
Magda Lúcia Félix de Oliveira
ORCID: https://orcid.org/0000-0003-4095-9382
Universidade Estadual do Maringá, Brasil
E-mail: magdauem @gmail.com
Jhennifer Galassi Bortoloci
ORCID: https://orcid.org/0000-0002-7807-8065
Universidade Estadual do Maringá, Brasil
E-mail: jhennifergbortoloci @ outlook.com
Cleiton José Santana
Faculdade Pitágoras, Brasil
ORCID: https://orcid.org/0000-0002-8150-2357
Fanail cleitonjsantana@ hotmail.com
E-maal:

\section{Resumo}

Objetivou-se descrever as internações por agrotóxicos registradas a um centro de informação e assistência toxicológica do Noroeste do Paraná, por meio de estudo transversal e retrospectivo, com dados da Relação de Pacientes Internados, do Centro de Controle de Intoxicações do Hospital Universitário Regional de Maringá. Foram identificados 170 indivíduos internados com intoxicação aguda ou crônica por agrotóxicos, nos meses de janeiro de 2016 a dezembro de 2020. Predominou a faixa etária de 15 a 45 anos - 117 casos (68,8\%), no entanto foram encontrados 22 casos de crianças $(12,9 \%)$ e 13 em idosos (7,6\%). O sexo masculino representou 104 casos $(61,2 \%)$. A tentativa de suicido aconteceu em 120 casos $(70,6 \%)$ e o acidente individual em $41(24,1 \%)$. Os agrotóxicos da classe toxicológica herbicida representaram 59 casos (35\%), seguidos dos inseticidas piretróides - 35 (21\%) e organofosforados - 25 (15\%), mas não foram informados agentes tóxicos em 16 casos (9\%). A média de internação hospitalar foi de $2,95 \%$ dias e cinco casos $(2,9 \%)$ foram internados em unidade de terapia intensiva. A taxa de óbito para o total de casos foi de 3,5\%, a maioria por suicídio. Os dados destacam a necessidade do desenvolvimento de estratégias preventivas para o enfrentamento da intoxicação por agrotóxicos e elaboração de intervenções para redução do número de internações, minimizando complicações e óbitos.

Palavras-chave: Exposição a praguicidas; Hospitalização; Vigilância em saúde pública; Estudos transversais; Sistemas de informação em saúde.

\begin{abstract}
The objective was to describe hospitalizations for pesticides recorded at a toxicological information and assistance center in the Northwest of Paraná, through a cross-sectional and retrospective study, with data from the List of Inpatients, from the Poison Control Center of the Regional University Hospital of Maringá. 170 individuals hospitalized with acute or chronic poisoning by pesticides were identified from January 2016 to December 2020 . The age group from 15 to 45 years predominated - 117 cases $(68.8 \%)$, however, 22 cases of children (12.9\%) and 13 in the elderly $(7.6 \%)$. Males represented 104 cases $(61.2 \%)$. The suicide attempt occurred in 120 cases $(70.6 \%)$ and the individual accident in $41(24.1 \%)$. Pesticides of the herbicide toxicological class represented 59 cases (35\%), followed by pyrethroid insecticides - $35(21 \%)$ and organophosphates - $25(15 \%)$, but no toxic agents were reported in 16 cases
\end{abstract}


(9\%). The mean hospital stay was $2.95 \%$ days and five cases $(2.9 \%)$ were admitted to the intensive care unit. The death rate for the total number of cases was $3.5 \%$, the majority by suicide. The data highlight the need to develop preventive strategies to deal with pesticide poisoning and the development of interventions to reduce the number of hospitalizations, minimizing complications and deaths.

Keywords: Exposure to pesticides; Hospitalization; Public health surveillance; Cross-sectional studies; Health information systems.

\section{Resumen}

El objetivo fue describir las internaciones por pesticidas registradas en un centro de información y asistencia toxicológica en el Noroeste de Paraná, a través de un estudio transversal y retrospectivo, con datos de la Lista de Pacientes Internados, del Centro de Control de Intoxicaciones del Hospital Regional Universitario de Maringa. Se identificaron 170 personas hospitalizadas con intoxicación aguda o crónica por plaguicidas de enero de 2016 a diciembre de 2020. Predominó el grupo de edad de 15 a 45 años - 117 casos (68,8\%), sin embargo, 22 casos de niños $(12,9 \%)$ y 13 en los ancianos $(7,6 \%)$. Los varones representaron 104 casos $(61,2 \%)$. El intento de suicidio ocurrió en 120 casos $(70,6 \%)$ y el accidente individual en $41(24,1 \%)$. Los plaguicidas de la clase toxicológica herbicida representaron 59 casos (35\%), seguidos de los insecticidas piretroides - 35 (21\%) y los organofosforados - 25 (15\%), pero no se reportaron agentes tóxicos en 16 casos (9\%). La estancia hospitalaria media fue de $2,95 \%$ días y cinco casos $(2,9 \%)$ ingresaron en la unidad de cuidados intensivos. La tasa de mortalidad para el total de casos fue del 3,5\%, la mayoría por suicidio. Los datos destacan la necesidad de desarrollar estrategias preventivas para hacer frente a la intoxicación por plaguicidas y el desarrollo de intervenciones para reducir el número de hospitalizaciones, minimizando las complicaciones y muertes.

Palabras clave: Exposición a plaguicidas; Hospitalización; Vigilancia de la salud pública; Estudios transversales; Sistemas de información en salud.

\section{Introdução}

O uso de produtos químicos na agricultura aumentou a partir da década de 1950, incorporado aos conceitos da Revolução Verde e pautado na mecanização, monocultura, plantas altamente selecionadas e uso de agrotóxicos (Mcintyre et al., 2009; Aitte, 2021). Agrotóxicos são substâncias químicas utilizadas para controle e destruição de pragas, classificados em herbicidas, inseticidas, fungicidas, bactericidas, acaricidas e rodenticidas, amplamente utilizados no combate de pragas e doenças vegetais para elevar a produtividade agrícola e na zona urbana, em uso doméstico (WHo, 2020; García-García et al., 2016; Van Den Berg et al., 2020).

Com a síntese de novas moléculas, com diferentes tipos de mecanismos de ação e toxicidade (El-Nahhal \& El-Nahhal, 2021), um grande volume de agrotóxicos foram colocados à disposição da agricultura, porém não houve a preocupação em controlar a utilização, com consequente exposição humana e do meio ambiente aos danos que estes produtos podem ocasionar (Lopes \& Albuquerque, 2018; Aitte, 2021). O uso global de agrotóxicos aumentou de forma constante, de 2,3 milhões de toneladas estimados em 1990 para 4,1 milhões de toneladas em 2016 (Fao, 2019).

Em 2008, o Brasil ultrapassou os Estados Unidos e assumiu a posição de maior consumidor mundial de agrotóxicos e, enquanto nos últimos dez anos o mercado de agrotóxicos cresceu 93\% no mundo, no Brasil esse crescimento foi de $190 \%$ (Carneiro et al., 2015; Belchior et al., 2017; Rigotto et al., 2014).

O uso de agrotóxicos no mundo e na agricultura brasileira é um problema de saúde pública, por contaminação no ambiente e em alimentos e pelas intoxicações humana decorrentes (Piccoli et al., 2019; Who, 2020; El-Nahhal \& El-Nahhal, 2021; Aitte, 2021). Devido à extensa disponibilidade comercial e uso irregular, são responsáveis por expressivos casos de intoxicações (Who, 2020).

Na década de 1990, a Organização Mundial da Saúde - OMS estimou que cerca de um milhão de intoxicações não intencionais por agrotóxicos ocorriam anualmente no mundo, levando aproximadamente a 20 mil mortes (Boedeker et al., 2020). Trinta anos depois, embora não exista um quadro real de intoxicação global por agrotóxicos, os casos de intoxicações por agrotóxicos tendem a ser maiores, pois em um número maior de pessoas são expostas aos agrotóxicos (Boedeker et al., 2020; Hough, 2021). 
As intoxicações agudas por agrotóxicos são ocorrências frequentes nos serviços de atenção às urgências e emergências nos países em desenvolvimento, resultando em internações hospitalares (Freitas \& Garibotti, 2020), e efeitos da intoxicação aguda ou os efeitos secundários, como doenças orgânicas e comorbidades psico-orgânicas, devem ser identificados para a construção de medidas para o enfrentamento destes agravos (Kim et al., 2017; Piccoli et al., 2019; Dos Santos et al., 2020). No entanto, dados de intoxicação por agrotóxicos encontram-se subnotificados e invisíveis nas estatísticas em saúde, pois a Organização Mundial de Saúde - OMS estima que para cada caso notificado existam outros 50 casos não registrados (Who, 2020).

Embora desde 2004 exista um processo de vigilância epidemiológica para mensuração da intoxicação por agrotóxicos na saúde da população brasileira, por meio de notificação compulsória de exposição a agentes tóxicos (Brasil, 2019), a magnitude e a complexidade do quadro epidemiológico por esses agravos podem não estar devidamente quantificados, e a subnotificação ou notificação irregular dificulta a prevenção e atenção à saúde, pesquisas, e notificações judiciais (Queiroz et al., 2019; Brasil, 2018). Outro fator preocupante é o preenchimento inadequado de fichas/formulários/declarações que alimentam os sistemas de informação, negligenciando informações relevantes para a vigilância epidemiológica (Queiroz et al., 2019; Taveira \& Albuquerque, 2018).

No ano de 2017 foram registrados no Sistema de Informação e Agravo de Notificação - SINAN 5.238 casos de intoxicações agudas por agrotóxicos no Brasil e a maior parte dos eventos se concentraram na região Sul e Sudeste do país. No entanto, embora o Paraná seja o segundo maior consumidor de agrotóxicos no Brasil, e esse consumo tende a ocasionar intoxicações agudas e crônicas nas populações expostas, a notificação de intoxicações não corresponde a essa realidade (Brasil, 2021; Paraná, 2018).

Neste contexto, na construção de um sistema de informação para vigilância das populações expostas aos agrotóxicos, os centros de informação e assistência toxicológica brasileiros são unidades sentinelas para vigilância epidemiológica de doenças e agravos decorrentes da intoxicação por diversos agentes e circunstâncias (Costa \& Alonzo, 2019). Logo, estudos que se dediquem a explorar essas informações a partir dos CIAT, com fontes de dados controladas, de maior confiabilidade e maior cobertura das notificações, podem auxiliar tomada de decisão de gestores de saúde e, consequentemente, a condução de políticas públicas (Queiroz et al., 2019; Neto et al., 2020).

O presente estudo explorou as ocorrências e notificações de casos de intoxicação por agrotóxicos em um Centro de Informação e Assistência Toxicológica - CIAT. Fundamenta-se na necessidade de construção de medidas de enfrentamento dos efeitos dos agrotóxicos, um problema que afeta milhões de pessoas em todas as faixas etárias, com enorme variedade de consequências biopsicossociais, familiares e individuais e elevada morbimortalidade e custos sociais e econômicos (Who, 2020; Belchior, 2017; Kim et al., 2017; Rani et al, 2021; Hough, 2021).

O objetivo foi descrever as internações por agrotóxicos registradas e acompanhada por um centro de assistência toxicológica no período de cinco anos.

\section{Metodologia}

Estudo quantitativo, descritivo e transversal, com análise documental e retrospectiva de registros de internações por agrotóxicos, arquivados em um centro de informação e assistência toxicológica, denominado Centro de Controle de Intoxicações do Hospital Universitário Regional de Maringá (CCI/HUM) (Gil, 2010; Capp \& Nienov, 2020; Almeida-Filho \& RouquayroL, 2015).

O CCI/HUM integra a Rede Nacional de Centros de Informação e Assistência Toxicológica - RENACIAT. É um serviço público que funciona em regime de plantão de $24 \mathrm{~h}$, em apoio às urgências toxicológicas, com ações para a população 
interna e externa ao HUM, e assistência remota e presencial, prioritariamente, para os 115 municípios da Macrorregional Noroeste de Saúde do Paraná, e unidade sentinela para vigilância epidemiológica das intoxicações (Costa \& Alonzo, 2019).

A população em estudo foi representada por indivíduos de ambos os sexos, independentemente da idade, com registro de caso confirmado de intoxicação aguda ou crônica por agrotóxicos e hospitalização mínima de um dia (24 horas), notificados ao CCI/HUM no período de janeiro de 2016 a dezembro de 2020.

A fonte de dados foi a Relação de Pacientes Internados. Essa Relação é uma listagem impressa do CCI/HUM, na qual, diariamente, são registrados todos os pacientes com intoxicação pelas diversas etiologias e circunstâncias, atendidos no Centro. A variável desfecho do estudo foi à prevalência de internações hospitalares por agrotóxicos, e as variáveis categóricas estudadas foram: data da internação, idade e sexo do paciente, classe toxicológica do agente e circunstância da intoxicação, unidade e duração da internação e desfecho do caso. Acrescentou-se o item ignorado/não informado às variáveis analisadas.

A coleta de dados ocorreu meses de junho e julho de 2021, por auditoria retrospectiva no conjunto da Relação dos Pacientes Internados, separados os casos segundo o agente da intoxicação, e compilados os casos de intoxicação por agrotóxicos do período em estudo (60 meses) em uma planilha Microsoft Office Excel 2019, por mês e ano.

A planilha era de preenchimento anual, composta de dois módulos - variáveis sociodemográficas dos pacientes e variáveis da internação e atendimento em serviço de saúde (dados quantitativos brutos). Foi realizada análise descritiva dos resultados, para a obtenção de gráficos e tabelas de frequência absoluta e porcentagem para as variáveis categóricas.

O estudo recebeu parecer favorável do Comitê de Ética em Pesquisa, com parecer número 4.010.048, CAAE: 30101620.6.0000.0104

\section{Resultados e Discussão}

Do total de 170 casos de hospitalização por agrotóxicos no período, foi registrado maior número de casos internados em 2018 - 56 casos $(32,9 \%)$.

A análise do perfil socioeconômico dos participantes da pesquisa mostrou os indivíduos mais vulneráveis à indicação de internação eram do sexo masculino, com 104 casos $(61,2 \%)$, e maior prevalência na faixa etária de 31 a 45 anos, com 64 casos (37,6\%). Crianças até 14 anos representaram 22 casos (12,9\%) e idosos 13 casos (7,6\%) (Tabela1).

A tentativa de suicídio predominou como circunstância para os internamentos por agrotóxicos - 120 casos $(70,6 \%)$. As intoxicações acidentais representaram 41 casos (24,1\%), a maioria em crianças e adolescentes - 22 casos $(12,9 \%)$ (Tabela1).

As intoxicações ocupacionais representaram cinco casos (2,9\%), sendo três do sexo masculino, a idade variou de 31 a 59 anos, e não foram registrados óbitos por essa circunstância. Outras circunstâncias, como acidente ambiental, acidente coletivo, violência/homicídio, foram responsáveis por quatro casos, com desfecho favorável em todos os casos (Tabela1).

Os tipos de exposição relatadas foram aguda única, com 164 (96,4\%) dos casos notificados e média de dias de internamento equivalente a 2,9 dias. A maioria evoluiu para a cura sem sequelas, mas ocorreram seis óbitos (3,5\%) (Tabela1).

A maioria dos casos foram notificados em unidades hospitalares. Dos 120 (70,6\%) indivíduos que tentaram suicídio, cinco $(2,9 \%)$ foram admitidos em serviços de terapia intensiva - UTI, com evolução para óbito e dos indivíduos que tiveram intoxicação acidental - $40(99,4 \%)$ tiveram desfecho favorável com alta hospitalar, e um caso $(0,6 \%)$ evoluiu para óbito. 


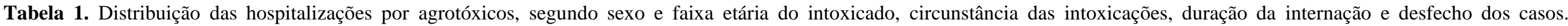
CCI/HUM, 2016 -2020. $(n=170)$.

\begin{tabular}{|c|c|c|c|c|c|c|c|c|c|}
\hline \multirow{3}{*}{ Variáveis } & \multicolumn{8}{|c|}{ Circunstância } & \multirow{3}{*}{ Total } \\
\hline & \multicolumn{2}{|c|}{$\begin{array}{c}\text { TS } \\
\mathrm{n}=120\end{array}$} & \multicolumn{2}{|c|}{$\begin{array}{c}\text { AI } \\
\mathrm{n}=41\end{array}$} & \multicolumn{2}{|c|}{$\begin{array}{c}\text { AO } \\
\mathrm{n}=5\end{array}$} & \multicolumn{2}{|c|}{$\begin{array}{c}\text { Outras } \\
n=4\end{array}$} & \\
\hline & $\begin{array}{c}\mathbf{F} \\
\mathrm{n}=49\end{array}$ & $\begin{array}{c}\mathbf{M} \\
\mathrm{n}=71\end{array}$ & $\begin{array}{c}\mathbf{F} \\
\mathrm{n}=14\end{array}$ & $\begin{array}{c}\mathbf{M} \\
\mathrm{n}=27\end{array}$ & $\begin{array}{c}\mathbf{F} \\
\mathrm{n}=2\end{array}$ & $\begin{array}{c}\mathbf{M} \\
\mathrm{n}=3\end{array}$ & $\begin{array}{c}\mathbf{F} \\
\mathrm{n}=1\end{array}$ & $\begin{array}{c}\mathbf{M} \\
\mathrm{n}=3\end{array}$ & \\
\hline \multicolumn{10}{|l|}{ Idade } \\
\hline $0-14$ anos & $0(0.00)$ & $1(1.41)$ & $10(71.43)$ & $11(40.74)$ & $0(0.00)$ & $0(0.00)$ & $0(0.00)$ & $0(0.00)$ & $22(12.94)$ \\
\hline $15-30$ anos & $19(38.78)$ & $28(39.44)$ & $2(14.29)$ & $3(11.11)$ & $0(0.00)$ & $1(33.33)$ & $0(0.00)$ & $0(0.00)$ & $53(31.18)$ \\
\hline $31-45$ anos & $20(40.82)$ & $30(42.25)$ & $0(0.00)$ & $7(25.93)$ & $2(100.00)$ & $2(66.67)$ & $1(100.00)$ & $2(66.67)$ & $64(37.65)$ \\
\hline $46-59$ anos & $7(14.29)$ & $8(11.27)$ & $0(0.00)$ & $2(7.41)$ & $0(0.00)$ & $0(0.00)$ & $0(0.00)$ & $1(33.33)$ & $18(10.59)$ \\
\hline $\begin{array}{l}60 \text { anos ou mais } \\
\text { Duração de } \\
\text { Internaçãa }\end{array}$ & $3(6.12)$ & $4(5.63)$ & $2(14.29)$ & $4(14.81)$ & $0(0.00)$ & $0(0.00)$ & $0(0.00)$ & $0(0.00)$ & $13(7.65)$ \\
\hline Média & 3,44 & 3,04 & 1,30 & 2,68 & 10,00 & 5,08 & 1,00 & 8,00 & 2,95 \\
\hline \multicolumn{10}{|l|}{ Desfecho } \\
\hline Alta & 48 (97.96) & 67 (94.37) & $13(92.86)$ & $27(100.00)$ & $2(100.00)$ & $3(100.00)$ & $1(100.00)$ & $3(100.00)$ & $164(96.47)$ \\
\hline Óbito & $1(2.04)$ & $4(5.63)$ & $1(7.14)$ & $0(0.00)$ & $0(0.00)$ & $0(0.00)$ & $0(0.00)$ & $0(0.00)$ & $6(3.53)$ \\
\hline
\end{tabular}

Legenda: Feminino (F) Masculino (M); Tentativa de suicídio (TS), Acidente individual (AI), Acidente ocupacional (AO). Fonte: Autores. 
Os agentes envolvidos nas intoxicações foram principalmente da classe toxicilógica herbicidas - 63 (35\%), incluindo o herbicida glifosato - 4\%, e inseticidas piretróides - 35 casos (21\%) e inseticidas organofosforados - 25 casos (15\%). Outros inseticidas representaram 17 casos (9\%) e a soma dos demais agentes, classificados como outros agrotóxicos - raticidas, fungicidas e acaricidas -, perfizeram um total de 11 casos de intoxicação (6\%). Não foram informadas a classificação toxicológica do agente, ou foram considerados ignorados ou indeterminados, em 16 casos (9\%) (Gráfico 1).

Gráfico 1. Distribuição das hospitalizações por agrotóxicos segundo a classificação toxicológica do agente. CCI/HUM, 2016 2020. $(n=170)$.

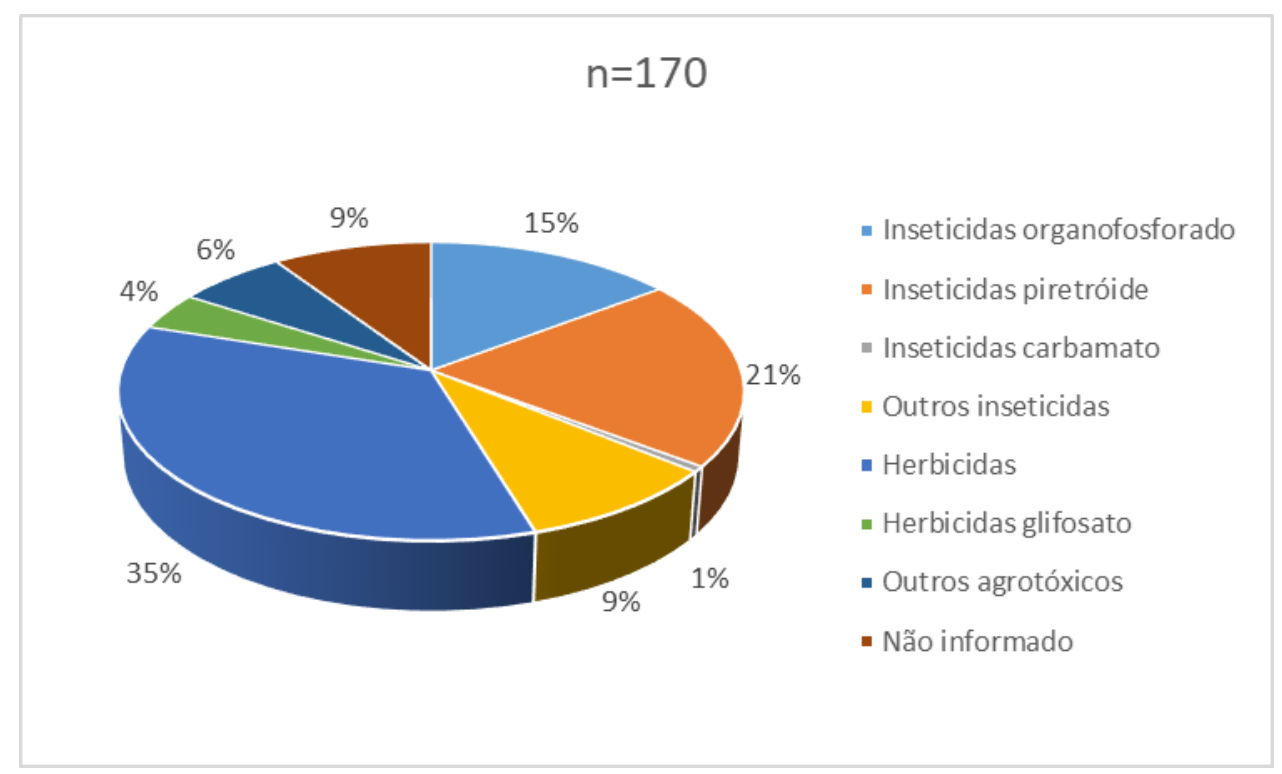

Fonte: CCI/HUM.

Dados do Sistema de Informação de Agravos de Notificação - SINAN indicam que as intoxicações aumentaram nos anos 2007 a 2016, e os agrotóxicos ocupavam a primeira posição de letalidade entre as intoxicações (Lopes \& Albuquerque, 2018). No ano de 2018, foram notificados 9914 casos de intoxicação por agrotóxicos de uso agrícola e doméstico no território brasileiro (Brasil, 2021).

De acordo com o Ministério da Saúde, 2014 foi o ano de maior incidência de casos de intoxicação por agrotóxicos, com registro de 6,26 casos em cada 100 mil habitantes. Os registros foram observados em todo o território nacional, com destaque para os estados do Paraná (7,69/100 mil hab.), Tocantins (6,48/100 mil hab.) e Espírito Santo (6,56/100 mil hab.) (Brasil, 2018). No entanto, a desatualização dos dados e a identificação de subnotificação no lançamento no SINAN confirmam a inconsistência de dados e a falta de comunicação entre os sistemas de informação em saúde brasileiros, resultando no ocultamento da real ocorrência de intoxicações por agrotóxicos (Queiroz et al., 2019; Taveira \& Albuquerque, 2018). Estudos em cenários locais apontam números superiores aos de base nacional (Tosetto, 2016; Bruehmueller et al., 2021; Magalhães \& Caldas, 2018; Martins, 2018).

Quanto às características de sexo e idade, os resultados do presente estudo corroboram achados da literatura nacional. Os intoxicados por agrotóxicos são homens em idade economicamente ativa, com fácil acesso aos agrotóxicos em seus ambientes de trabalho ou doméstico (Freitas \& Garibotti, 2020; Tosetto, 2016; Bruehmueller et al., 2021; Martins, 2018; Santos et al., 2021). No entanto, considerando que uma população é considerada exposta a agrotóxicos em uma rota de exposição que estabeleça contato do agrotóxico com a população receptora a partir de condições ambientais, laborais, 
acidentais e/ou intencionais, (Rani et al., 2021; Hendges et al., 2019), crianças, em acidentes domésticos, e idosos também são expostos ao risco de intoxicação.

As implicações dos agrotóxicos para a saúde humana não se restringem apenas à exposição na produção agrícola ou pecuária. A intoxicação por agrotóxicos configura-se, nas últimas décadas, como problema rural e urbano, ou seja, os agrotóxicos representam um importante problema para a saúde humana, tanto no meio rural, quanto no meio urbano (Robert \& Reigart, 2013). Também, as intoxicações não são reflexos de uma relação simples entre produto e pessoa exposta, fatores relativos às condições de exposição ou do trabalho e o fato de ter ocorrido exposição/contato em um único tipo de produto ou a vários deles são determinantes (Rani et al, 2021).

Principalmente para pessoas que vivem próximas ou em áreas agrícolas, os agrotóxicos representam um problema ocupacional, como uma das consequências do uso indiscriminado na zona rural (Brasil, 2018). Em relação à internação decorrente da exposição ocupacional, o número de casos encontrados foi pouco expressivo, mas isso pode ocorrer pela dificuldade tanto de acesso ao sistema de saúde quanto de entender os sinais e sintomas da intoxicação como tais, por serem similares aos de problemas de saúde comuns como vertigens, cefaleia e fadiga, e a intoxicação crônica e ocupacional por agrotóxicos pode apresentar-se de forma "silenciosa" (Ubessi et al., 2015; Santos et al., 2021).

Resultados do estudo desenvolvido por Viero et al. (2016) apontaram que o trabalhador rural tem conhecimento de que há riscos relacionados ao manuseio de agrotóxicos, mas, em geral, negam associação direta entre o uso de agrotóxicos e problemas de saúde, pois evidenciaria que não utilizam adequadamente equipamentos de proteção individual. Essa subnotificação diminui a compreensão do risco dos agrotóxicos e da incidência e gravidade das intoxicações em trabalhadores rurais.

No meio urbano, o mesmo panorama é vivenciado, visto que agrotóxicos são utilizados em uso doméstico e em campanhas de saúde pública (Carneiro et al., 2015; Roberts \& Reigart, 2013;). De acordo com o Atlas de Geografia do Uso de Agrotóxicos no Brasil e Conexões com a União Europeia (BOMBARDI, 2018), para a extensa exposição ocupacional e ambiental aos agrotóxicos, com baixa notificação, ocorrem também casos acidentais e intencionais associados ao contato ou à ingestão desses produtos.

A auto-intoxicação por agrotóxicos representa 110.000-168.000 (14-20\%) dos suicídios globais (Mew et al., 2017; Who, 2020), e é particularmente comum em países de baixa e média renda, onde a agricultura em pequena escala permite fácil acesso a agrotóxicos altamente perigosos entre famílias e comunidades. Mortes intencionais com agrotóxicos estão entre as cinco principais causas de suicídios em todo o mundo, contribuindo com indicadores de mortalidade nos países em desenvolvimento (Fao, 2019; Mew et. al., 2017).

A gravidade das intoxicações não é reflexo de uma relação simples entre o produto e a pessoa exposta. Vários fatores participam de sua determinação, dentre eles as condições de exposição ou do trabalho e a exposição/contato em um único tipo de produto ou a vários deles (Carneiro et al., 2015; Who, 2020; Van Den Berg, et al., 2020). Os pacientes que se intoxicaram acidentalmente e os que tentaram suicídio apresentaram complicações de saúde mais severas, demandando assistência à saúde hospitalar.

Com a síntese de novas moléculas, com diferentes tipos de mecanismos de ação e toxicidade (El-Nahhal \& El-Nahhal, 2021), os agrotóxicos impactam cada vez mais a saúde humana, produzindo efeitos que variam conforme o princípio ativo, a dose absorvida e a forma de exposição. Essa toxicidade depende das propriedades dos ingredientes ativos e coadjuvantes do produto, podendo levar a óbito (Tosetto et al., 2021).

A Organização Mundial da Saúde, por meio do Intergovernamental Forum on Chemical Safely (IFCS), publicou em 2008 uma proposta de classificação para as intoxicações agudas por agrotóxicos. A padronização dos critérios que envolvem a 
classificação desses agravos à saúde favorece o registro dos dados epidemiológicos e, consequentemente, a possibilidade de comparação dos dados de registro tanto entre diferentes regiões como ao longo do tempo (Thundiyl et al., 2008).

As intoxicações podem ser agudas ou crônicas. Intoxicação aguda é a que ocasiona alteração no estado de saúde de um ou mais indivíduos, geralmente única e ocorre no período de até 24 horas após a exposição ao agente tóxico, podendo ocorrer de forma leve, moderada ou grave, dependendo do tempo de absorção e da toxicidade do produto. Manifesta-se por meio de um conjunto de sinais e sintomas que podem ocorrer de forma súbita, imediatamente ou horas após a exposição (Tosetto et al., 2021). Esse tipo de intoxicação foi predominante no presente estudo.

No entanto, a análise dos dados evidenciou que há um predomínio de casos de intoxicação por agrotóxicos da classe toxicológica herbicida, referidos na literatura como possíveis agentes de agravos crônicos não transmissíveis, como neoplasias e problemas neurocomportamentais (Eddleston, 2020). Nessa condição, passam despercebidas intoxicações por produtos como o glifosato, responsável pela metade dos agrotóxicos consumidos no Brasil, classificado como de baixa toxidade aguda, mas podem causar danos graves e irreversíveis à saúde (Karunarathne et al., 2020).

De acordo com a Organização Mundial da Saúde, a estimativa da taxa de fatalidade de pessoas que realizaram o uso de herbicidas para suicídio foi de $43 \%$ em 2017 (Who 2019). Estudos ao redor do mundo comprovam que ao proibir o herbicida de comercialização e uso na agricultura, casos de tentativa de suicídio e mortes por este motivo diminuíram (Arya et al., 2019; Boucaud-Maitre et al., 2019; Bowles,1995).

Embora a literatura nacional indique os inseticidas organofosforados e carbamatos, inibidores das enzimas colinesterases, como principais agrotóxicos envolvidos em intoxicações graves no Brasil (Klein et al., 2018), neste estudo foi encontrado superioridade do herbicida, individualmente, como agente que resultou em internação hospitalar.

A taxa de mortalidade encontrada no estudo foi similar a de países desenvolvidos, um indicador de que o manejo dos pacientes intoxicados com agrotóxicos tem sido apropriado, porém ressalta-se a gravidade destas ocorrências e a utilização de leitos de terapia intensiva, principalmente entre os pacientes que tentaram suicídio (Fao, 2019; Mew et. al., 2017).

Estudo realizado nos anos 2000-2009, indicou a queda da mortalidade de intoxicação ocupacional por agrotóxicos no período, exceto no Nordeste, e maior entre os homens que em mulheres. A maior parte dos óbitos foi causada por agrotóxicos do tipo inseticidas organofosforados e carbamatos (Sousa et al., 2013).

Nos anos 2010 e 2017, estudo realizado em um Instituto Médico Legal encontrou registro de número de casos de óbitos por agrotóxicos para ambos os sexos - 15 casos femininos e 17 casos masculinos. A faixa etária compreendida entre 20 e 49 anos representou a maioria dos casos, e os extremos de idade estiveram presentes em um caso de criança e de um idoso, com 80 anos. As circunstâncias registradas nos laudos periciais eram mortes decorrente de suicídio ou acidental. Os agrotóxicos da classe dos herbicidas foram os mais referidos nos históricos das intoxicações que acompanhavam o óbito herbicida, mata- mato, glifosato -, contudo apenas os agrotóxicos da classe dos inseticidas foram identificados por analise toxicológica $\mathrm{O}$ trabalho agrícola não foi apontado como fator determinante para morte por intoxicações (Bruehmueller et al., 2021).

A frequência, o uso indevido e a gravidade das intoxicações apontam a necessidade de medidas que visem à restrição e o acesso indiscriminado aos agrotóxicos, conscientizar a importância do uso de equipamentos de proteção individual, instituir medidas de toxicovigilância e reconhecer precocemente estes agravos à saúde.

\section{Conclusão}

A limitação do estudo está relacionada à análise de dados secundários de um cenário regional, mas os resultados apontam a realidade das internações por agrotóxicos, consideradas como indicadoras de gravidade, registradas em um centro de informação e assistência toxicológica, considerado unidade sentinela para vigilância epidemiológica das intoxicações. 
Também, dados locais e regionais são necessários para caracterizar o perfil de risco e vulnerabilidade de grupos e apoiar programas de intervenção de saúde locais.

A análise dos dados evidenciou um predomínio de casos de intoxicação por agrotóxicos pela classe toxicológica herbicida, em adultos jovens do sexo masculino, e a principal circunstância das internações foi a tentativa de suicídio. A maioria evoluiu para cura sem sequelas, mas a taxa de mortalidade encontrada foi de 3,5\%, predominantemente por ação intencional. A internação em unidade de terapia intensiva e a mortalidade indicaram gravidade dos casos

A incidência de intoxicações moderadas/graves e a mortalidade por agrotóxicos sugerem estratégias preventivas robustas frente à utilização destes produtos, com os objetivos de controle da comercialização e restrição ao acesso indiscriminado. Os dados destacam a necessidade do desenvolvimento de estratégias preventivas para o enfrentamento das intoxicações por agrotóxicos, elaboração de intervenções para redução do número de internações, minimizando complicações e óbitos. Nesse sentido, considera-se que este estudo possibilitou o levantamento de questões importantes, relacionadas à internação hospitalar por agrotóxicos, e a divulgação dos dados poderão auxiliar na formulação da Política de Agrotóxicos no Brasil.

\section{Referências}

Aitte, S. (2021). Review the impacts of pesticides on the environmental health and human Health. African Journal of Aquatic Science 12(3). https://www.researchgate.net/publication/355911508_Review_the_impacts_of_pesticides_on_the_environmental_health_and_human_Health.

Almeida Filho, N., \& Rouquayrol, M. Z. (2015). Introdução a Epidemiologia. Guanabara Koogan (4a ed.).

Arya, V., Page, A., Gunnell, D., Dandona, R., Mannan, H., Eddleston, M., \& Armstrong, G. (2019). Suicide by hanging is a priority for suicide prevention: method specific suicide in India (2001-2014). Journal of Affective Disorders, 257, 1-9. https://doi.org/10.1016/J.JAD.2019.07.005.

Belchior, D. C. V., Saraiva, A. de S., López, A. M. C., \& Scheidt, G. N. (2017). Impactos de Agrotóxicos no Meio Ambiente. Cadernos de Ciência \& Tecnologia, Brasília, 34 (1), 135-151. https://ainfo.cnptia.embrapa.br/digital/bitstream/item/164063/1/Impactos-de-agrotoxicos-sobre-o-meio-ambiente.pdf.

Boedeker, W., Watts, M., Clausing, P., \& Marquez, E. (2020). The global distribution of acute unintentional pesticide poisoning: estimations based on a systematic review. In BMC Public Health. 20(1). https://doi.org/10.1186/s12889-020-09939-0.

Bombardi, L.M. (2018). Geografia do Uso de Agrotóxicos no Brasil e Conexões com a União Europeia. FFLCH - USP, 296.

Boucaud-Maitre, D., Rambourg, M. O., Sinno-Tellier, S., Puskarczyk, E., Pineau, X., Kammerer, M., Bloch, J., \& Langrand, J. (2019). Human exposure to banned pesticides reported to the French Poison Control Centers: 2012-2016. Environmental toxicology and pharmacology, 69, 51-56. https://doi.org/10.1016/j.etap.2019.03.017.

Bowles, Jr. (1995). Suicide in Western Samoa: an example of a suicide prevention program in a developing country. In: Diekstra RFW, et al., editors. Preventative strategies of suicide. Leiden: E J Brill, 173-206.

Brasil. Departamento de Informática do Sistema Único De Saúde (DATASUS). (2021). Intoxicação Exógena - Notificações registradas no SINAN NET Brasil. http://tabnet.datasus.gov.br/cgi/tabcgi.exe?sinannet/cnv/Intoxbr.def.

BRASIL. Ministério da Saúde. (2018). Secretaria de Vigilância em Saúde. Departamento de Vigilância em Saúde Ambiental e Saúde do Trabalhador. Agrotóxicos na ótica do Sistema Único de Saúde. Brasília: Ministério da Saúde.

BRASIL. Ministério da Saúde. (2019). Secretaria de Vigilância em Saúde. Guia de vigilância em saúde. Brasília: Ministério da Saúde. 3. ed. https://portalarquivos2.saude.gov.br/images/pdf/2019/junho/25/guia-vigilancia-saude-volume-unico-3ed.pdf.

Bruehmueller, A., Moraes, T., Damasceno, D. A., Fernandes, V., \& Almeida, C. (2021). Ocorrência de Óbitos por Agrotóxicos no IML de Cuiabá , entre 2010 e 2017 Deaths Ocurrence by Pesticides in Cuiaba IML between 2010 and 2017. Ensaio \& Ciência, 25(3), 315-321.

Capp, E., \& Nienov, O. H. (2020). Epidemiologia aplicada básica. UFRGS.

Carneiro, F. F., Rigotto, R. M., Augusto, L. G. S., Friedrich, K., \& Búrigo, A. C. (2015). Dossiê ABRASCO: um alerta sobre os impactos dos agrotóxicos na saúde. Rio de Janeiro: EPSJV, São Paulo: Expressão Popular.

Costa, A. de O., \& Alonzo, H. G. A. (2019). Centros de Informação e Assistência Toxicológica no Brasil: descrição preliminar sobre sua organização e funções. Saúde Em Debate, 43(120), 110-121. https://doi.org/10.1590/0103-1104201912008.

Dos Santos, J. C. P., Valli, J. B., Sesse, N. S., Mackenzie-Ross, S., Zandonade, E., Ayres, L. R., \& Sampaio, K. N. (2020). Sociodemographic characteristics and exposure patterns of pesticide-related cases reported to a poison service center in Brazil between 2012 and 2016. Archives of Environmental \& Occupational Health, 76(8), 494-503. https://doi.org/10.1080/19338244.2020.1848773. 
El-Nahhal, I., \& El-Nahhal, Y. (2021). Pesticide residues in drinking water, their potential risk to human health and removal options. Journal of Environmental Management, 299, 113611. https://doi.org/10.1016/j.jenvman.2021.113611.

Fao. Food and Agriculture Organization. (2019). Preventing suicide: a resource for pesticide registrars and regulators. https://apps.who.int/iris/bitstream/handle/10665/326947/9789241516389-eng.pdf.

Freitas, A. B. de, \& Garibotti, V. (2020). Characterization of notifications of exogenous pesticide poisoning in Rio Grande do Sul, Brazil, 2011-2018. Epidemiologia e Serviços de Saúde, 29 (5), e2020061. https://doi.org/10.1590/S1679-49742020000500009.

García-García, C. R., Parrón, T., Requena, M., Alarcón, R., Tsatsakis, A. M., \& Hernández, A. F. (2016). Occupational pesticide exposure and adverse health effects at the clinical, hematological and biochemical level. Life Sciences, 145, 274-283. https://doi.org/10.1016/j.lfs.2015.10.013.

Gil, A. C. (2010). Como elaborar projetos de pesquisa. 5. ed. São Paulo: Atlas.

Hendges, C., Schiller, A. P., Manfrin, J., Macedo-JR, E. K., Gonçalves-JR, A. C., \& Stangarlin, J. R. (2019). Intoxicação humana por agroquímicos na região do Sul do Brasil entre 1999 e 2014. Journal of Environmental Science and Health, Part B. 54(4),219-225.

Hough, R. L. (2021). A world view of pesticides. Nature Geoscience, 14 (4), 183-184. http://portalsaude.saude.gov.br/index.

Iaastd. (2009). International Assessment of Agricultural Knowledge, Science and Technology for Development. Agriculture at a Crossroads, Global Report Chs 1, (4). http://www.fao.org/fileadmin/templates/est/Investment/Agriculture_at_a_Crossroads_Global_Report_IAASTD.pdf. www.islandpress.org.

Karunarathne, A., Gunnell, D., Konradsen, F., \& Eddleston, M. (2019). Clinical Toxicology How many premature deaths from pesticide suicide have occurred since the agricultural Green Revolution? How many premature deaths from pesticide suicide have occurred since the agricultural Green Revolution? https://doi.org/10.1080/15563650.2019.1662433.

Kim, K. H., Kabir, E., \& Jahan, S. A. (2017). Exposure to pesticides and the associated human health effects. Science of The Total Environment, 575, 525535. https://doi.org/10.1016/J.SCITOTENV.2016.09.009.

Klein, B., Staudt, K., Missio, R., Peruzzi Hammad, M., \& Almeida Alves, I. (2018). Análise do impacto do uso de organofosforados e carbamatos em trabalhadores rurais de um município da região noroeste do estado do Rio Grande do Sul. Acta Toxicológica Argentina, 26 (3), 104-112.

Lopes, C. V. A., \& Albuquerque, G. S. C. de. (2018). Agrotóxicos e seus impactos na saúde humana e ambiental: uma revisão sistemática. Saúde Em Debate, 42 (117), 518-534. https://doi.org/10.1590/0103-1104201811714.

Magalhães, A. F. A., \& Caldas, E. D. (2018). Underreporting of fatal poisonings in Brazil - A descriptive study using data from four information systems. Forensic Science International, 287, 136-141. https://doi.org/10.1016/J.FORSCIINT.2018.03.040

Martins, G. F. (2018). Relatório: Vigilância em saúde de populações exposta a agrotóxicos no Estado do Paraná. Brasília: Ministério da Saúde

Mcintyre, B. D., Herren, H. R., Wakhungu, J., \& Watson, R.T. (2009). Agriculture at a crossroads: global report. Washington DC: International Assessment of Agricultural Knowledge, Science and Technology for Development.

Mew, E. J., Padmanathan, P., Konradsen, F., Eddleston, M., Chang, S. Sen, Phillips, M. R., \& Gunnell, D. (2017). The global burden of fatal self-poisoning with pesticides 2006-15: Systematic review. Journal of Affective Disorders, 219, 93-104. https://doi.org/10.1016/J.JAD.2017.05.002

Neto, J. H. V. D. S., Coutinho, K., Valentim, R., Neto, C. G., Cardoso, P., Melo, A., \& Sidrim, M. (2020). Revista Brasileira de Inovação Tecnológica Em Saúde. 10 (1), 17-17. https://doi.org/10.18816/R-BITS.V10I1.19256.

Paraná. Secretaria de Estado da Saúde do Paraná. (2018). Superintendência de Atenção à Saúde. Linha Guia da Atenção às Populações Expostas aos Agrotóxicos - 1 ed. - Curitiba: SESA, 72.

Piccoli, C., Cremonese, C., Koifman, R., Koifman, S., \& Freire, C. (2019). Occupational exposure to pesticides and hematological alterations: A survey of farm residents in the South of Brazil. Ciência \& Saúde Coletiva, 24 (6), 2325-2340. https://doi.org/10.1590/1413-81232018246.13142017.

Queiroz, R. P., Lima, K. C., Carneiro De Oliveira, T., Marques, M., Ii, S., Ferreira, J., Iii, J., Maria, A., \& Mendes De Oliveira, B. (2019). Notifiable Diseases Information System and human poisoning by pesticides in Brazil Sistema de Informação de Agravos de Notificação e as intoxicações humanas por agrotóxicos no Brasil. https://doi.org/10.1590/1980-549720190033.

Rani, L., Thapa, K., Kanojia, N., Sharma, N., Singh, S., Grewal, A. S., Srivastav, A. L., \& Kaushal, J. (2021). An extensive review on the consequences of chemical pesticides on human health and environment. Journal of Cleaner Production, 283, 124657. https://doi.org/10.1016/J.JCLEPRO.2020.124657.

Rigotto, M. R., Vasconcelos, D. P., \& Rocha, M. M. (2014). Pesticide use in Brazil and problems for public health. Cad Saúde Pública. 30 (7), $1360-1362$. https://doi.org/10.1590/0102-311XPE020714.

Roberts, J. R., \& Reigart, J. R. (2013). Paraquat and diquat. In recognition and management of pesticide poisonings Office of Pesticide Programs U.S. Environmental Protection Agency, 6th ed, 110-116.

Santos, I. N., Santos Ferraz, I., Kelle, L., Lirio, S., Soares Da Silva, A., De, G., Sotero, S., De, G., \& Ruela, A. (2021). Implicações das intoxicações exógenas por agrotóxicos à saúde do trabalhador: uma revisão integrativa. Revista Ibero-Americana de Humanidades, Ciências e Educação, 7 (2), 16. https://doi.org/10.51891/rease.v7i2.582

Sousa, V., Maria, S. I., Peres Moura, C., Ferreira, F., Ii, N., \& Santana, V. S. (2013). Mortalidade por intoxicação ocupacional relacionada a agrotóxicos, 2000-2009, Brasil. Revista de Saúde Pública, 47 (3), 598-606. https://doi.org/10.1590/S0034-8910.2013047004306 
Research, Society and Development, v. 11, n. 2, e16511326318, 2022

(CC BY 4.0) | ISSN 2525-3409 | DOI: http://dx.doi.org/10.33448/rsd-v11i3.26318

Taveira, B. L. S., \& Albuquerque, G. S. C. de. (2018). Análise das notificações de intoxicações agudas, por agrotóxicos, em 38 municípios do estado do Paraná. Saúde Em Debate, 42(4), 211-222. https://doi.org/10.1590/0103-11042018s417.

ThundiyL, J. G., Stober, J., Besbelli., \& Pronczuk, J. (2008). Acute pesticide poisoning: a proposed classification tool. Bull World Health Organ, 86 (3), $205-$ 209.

Tosetto, E. E. (2016). Identificação das causas das subnotificações das intoxicações da população exposta a agrotóxicos: estudo de caso de Laranjeiras do Sul PR. https://rd.uffs.edu.br:8443/handle/prefix/605

Ubessi, L. D., Ubessi, C., Kirchner, R. M., Jardim, V. R., \& Stumm, E. M. F. (2015). Artigo Original Uso De Equipamentos De Proteção Por Agricultores Que Utilizam. 9 (4). https://doi.org/10.5205/reuol.7275-62744-1-SM.0904201507.

Van Den Berg, H., Gu, B., Grenier, B., Kohlschmid, E., Al-Eryani, S., da Silva Bezerra, H. S., Nagpal, B. N., Chanda, E., Gasimov, E., Velayudhan, R., \& Yadav, R. S. (2020). Pesticide lifecycle management in agriculture and public health: Where are the gaps? Science of the Total Environment, $742,140598$. https://doi.org/10.1016/j.scitotenv.2020.140598.

Viero, C. M., Camponogara, S., Regina Cezar-Vaz, M., Zavarese da Costa, V., Lúcia Colomé Beck, C., \& Anna, E. (2016). Risk society: the use of pesticides and implications for the health of rural workers. Escola Anna Nery, 20 (1), 99-105. https://doi.org/10.5935/1414-8145.20160014

WHO. Food and Agriculture Organization (FAO). Preventing suicide: a resource for pesticide registrars and regulators. Geneva, 2019. https://apps.who.int/iris/bitstream/handle/10665/326947/9789241516389-eng.pdf

WHO. The WHO Recommended Classification of Pesticides by Hazard and Guidelines to Classification 2009. Germany, 2010. https://apps.who.int/iris/bitstream/handle/10665/44271/9789241547963_eng.pdf?sequence=1\&isAllowed=y.

WHO. World Health Organization. Bulletin of the World Health Organization, (2020). https://www.who.int/bulletin/volumes/86/3/07-041814/en/. 